\title{
Surface-Potential-Based Compact Model for Quantum Effects in Planar and Double-Gate MOSFET
}

\author{
A.Yu. Serov, S.-M. Hong, Y.J. Park and H.S. Min \\ School of EECS and NSI-NCRC, Seoul National University, Seoul 151-744, Korea \\ andrew@isis.snu.ac.kr
}

\begin{abstract}
The surface-potential-based compact model for quantum effects in planar and doublegate MOSFETs is developed. The surface-potentials at source and drain sides are calculated with the effective field approximation and the quantum charge epxression. The drain current is calculated with the drift-diffusion model and quantum correction of the lateral field. One set of equations is used for both planar and double-gate structures.
\end{abstract}

\section{Introduction}

The compact modeling of quantum effects in MOSFET was originally performed with effective capacitance or effective band-gap techniques $[1,2]$. In order to have more accurate solution, the MOS equation has been modified to include the quantization in the channel of doublegate and planar MOSFET [3, 4].

However, the solution in [4] for planar MOSFET assumes the surface field as a confining potential, which does not allow to obtain good agreement with the SchrödingerPoisson simulation in the strong inversion condition. So, the factor of 0.5 for role of electron charges in expression of effective field should be added, as it is done in [5]. Using the effective-field approximation together with bulk-quantization correction term we obtain the MOS equation, which should be solved numerically in one iteration loop in order to find the surface potentials at source and drain sides for both planar and double-gate MOSFET structures.

In order to calculate the drain current, we use the drift diffusion model, where the lateral field is determined as the gradient of the lowest channel subband. It allows us to obtain the analytical expression for quantum correction to the conventional charge-sheet expression, developed in [6]. We compare our results with the Schrödinger-Poisson scheme, and modeling results for drain current are compared with the density-gradient simulation [7].

\section{Model}

Surface-potentials are calculated by solving the MOS equation with the quantum charge, which, in planar case, leads to

$$
\begin{aligned}
& C_{o x}\left(V_{g}-V_{F B}-\psi_{s}\right)=Q_{n}+Q_{d}, \\
& Q_{n}=q \sum_{i} \sum_{j=1}^{3} \frac{m_{x y}^{i}}{\pi \hbar^{2}} k_{B} T \log \left(\exp \left(\frac{E_{f}-E_{j}^{i}}{k_{B} T}\right)+1\right),
\end{aligned}
$$


where $i$ denotes the valley's index, $m_{x y}=\sqrt{m_{x} m_{y}}$ is the two-dimensional DOS effective mass, while z-axis is directed to the oxide interface, $j$ is the subband index, $E_{f}$ and $E_{j}$ are the Fermi level and quantization energy levels in the channel, respectively.

The energy spectrum in planar structure can be calculated with the triangular potential approximation, where the effective field is determined as

$$
E_{e f f}=\frac{\alpha Q_{n}+Q_{d}}{\varepsilon_{s i}}=\alpha C_{o x} \frac{V_{g}-V_{F B}-\psi_{s}}{\varepsilon_{s i}}+\frac{1-\alpha}{\varepsilon_{s i}} Q_{d}\left(\psi_{s}\right),
$$

where $\alpha$ is the fitting parameter, which is equal to 0.5 as a nominal value.

Then the subbands in planar MOSFET are determined as

$$
E_{S G, j}^{i}=-q \psi_{s}+\left(\frac{\hbar^{2}}{2 m_{z}^{i}}\right)^{\frac{1}{3}}\left[\frac{3}{2} \pi q E_{e f f}\left(j-\frac{1}{4}\right)\right]^{\frac{2}{3}}
$$

where the intrinsic energy in the substrate is the reference point. Since the effective field is the function of $\psi_{s}$ and $V_{g}$ we can solve the MOS equation for planar structure iteratively.

The effective field in double-gate structure can be defined in the similar manner, and we use the energy spectrum for two lowest energy levels as

$$
E_{D G, j}^{i}\left(\psi_{s}, V_{g}\right)=-q \psi_{s}+\frac{\hbar^{2} \pi^{2}}{8 m_{z}^{i} d^{2}} j^{2}+\left(\frac{\hbar^{2}}{2 m_{z}^{i}}\right)^{\frac{1}{3}}\left[\frac{9}{8} \pi q E_{e f f}\right]^{\frac{2}{3}} .
$$

In the subthreshold regime, the bulk component of the spectrum is dominated, whereas, the surface quantization part has the major effect in the deep inversion region. We will check the validity of our model in weak inversion by comparison with the SchrödingerPoisson solver.

Once the surface potential is determined, the gate current density can be calculated analytically using the effective field (3) and semi-classical approach [8].

We derive the drain current using the drift-diffusion model, where the lateral field is determined with as a gradient of the lowest subband along the channel

$$
I_{d s}=\mu Q_{n} \frac{1}{q} \frac{E_{0}}{d y}+\mu \frac{k T}{q} \frac{d Q_{n}}{d y},
$$

where $E_{0}$ is the lowest subband.

Integrating (6) for the double-gate case we obtain

$$
\begin{array}{r}
I_{d s} \frac{L_{c h}}{\mu_{0}}=\frac{2 C_{o x}}{q}\left(V_{g}-V_{F B}-\frac{Q_{d}}{C_{o x}}\right)\left[E_{S L}-E_{S 0}\right] \\
+\frac{k T}{q} 2 C_{o x}\left(\psi_{S L}-\psi_{S 0}\right)-C_{o x}\left(\psi_{S L}^{2}-\psi_{S 0}^{2}\right)-2 C_{o x} \int_{\psi_{S 0}}^{\psi_{S L}} d \psi_{s}\left(\frac{1}{q} \frac{d E_{0}}{d \psi_{s}}+1\right) \psi_{s}
\end{array}
$$

where $E_{S 0}, E_{S L}$ are the lowest subbands at source and drain sides. Integral in (7) can be calculated analytically without using special functions. The similar analytical expression for the planar MOSFET can also be derived. If we omit the lateral field correction, the expression (7) becomes the same as the conventional charge-sheet model [6]. 


\section{Simulation Results}

We compare the simulation results for MOS structures with the Schrödinger-Poisson scheme, which includes the wave function penetration into oxide region [11]. The C-V characteristics for planar structure are shown in Fig.1(a), where the substrate doping is $N_{a}=5 \cdot 10^{18} \mathrm{~cm}^{-3}$. Since we assumed the surface-quantization conditions in inversion of DGFET, our model should show better results for thick structures, where bulk quantization is negligible and device behavior approaches the planar limit. For validation purpose we simulated the thin structure and C-V dependencies for DGMOS with $5 \mathrm{~nm}$ silicon thickness and instrinsic channel as shown in Fig.1(b). The agreement between two models is worse in the weak inversion, but looks reasonable for higher bias. The corresponding gate tunneling current densities are shown on Fig.1(c) and Fig.1(d). In calculating tunneling current for the double-gate case, the tunneling barrier hitting frequency in the bulk quantization was assumed.

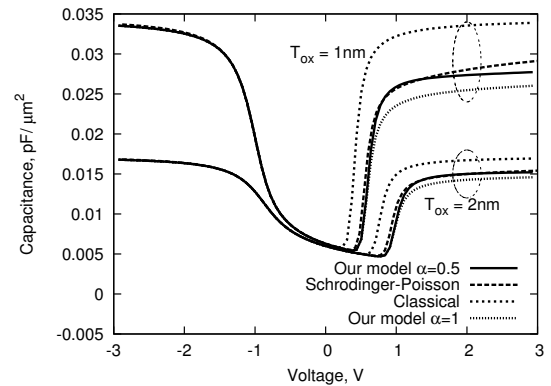

(a) CV in planar MOS

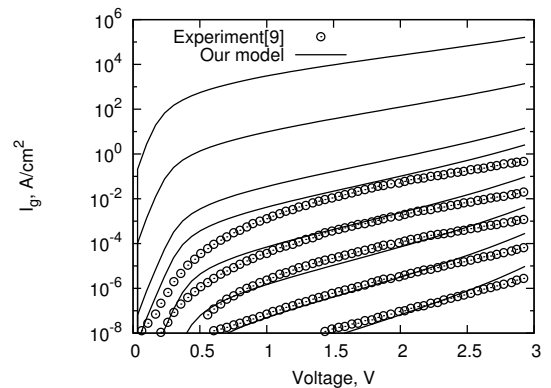

(c) Tunneling current in planar MOS

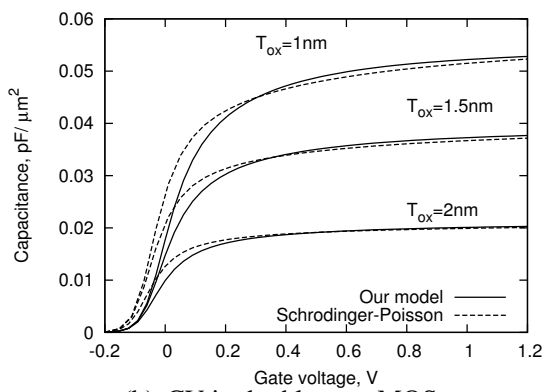

(b) $\mathrm{CV}$ in double-gate MOS

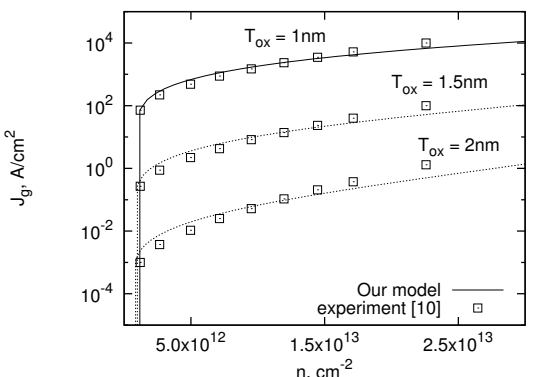

(d) Tunneling current in double-gate MOS

Figure 1: The dependence of capacitance on gate voltage for (a) planar structure, (b) double-gate structure. Tunneling current density for (c) planar and (d) double-gate MOS

Then we compare the modeling results for the drain current of long-channel MOSFET with the data from the density-gradient method with constant mobility [7].

We also compare our results with the model without the correction for lateral field. It can be observed in Fig.2, that the model with lateral field quantum correction predicts higher current and shows better agreement with density-gradient simulation. Since our model uses the similar framework with HiSIM model [12], the further modifications for short-channel effects can be made in straightforward manner under HiSIM framework. 


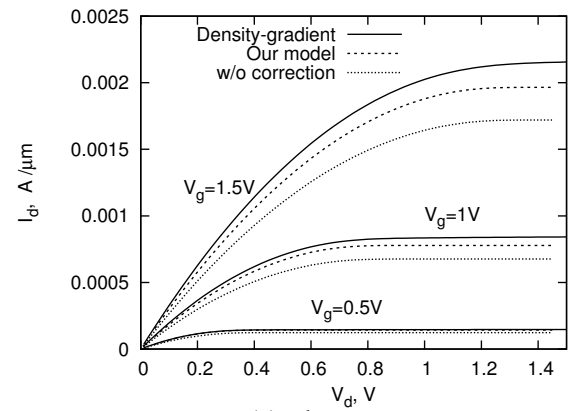

(a) planar

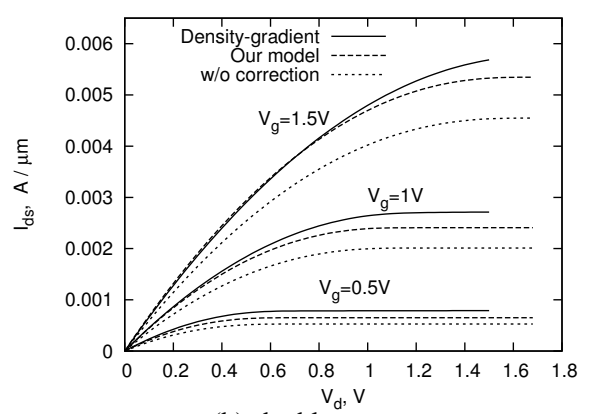

(b) double-gate

Figure 2: The drain current as a function of drain voltage for different gate voltages. The planar structure has $10 \AA$ oxide and $N_{a}=8 \cdot 10^{17} \mathrm{~cm}^{-3}$. The double-gate structure with channel thickness $5 \mathrm{~nm}$ and oxide thickness $1 \mathrm{~nm}$. Channel length is $1 \mu \mathrm{m}$.

\section{Conclusions}

The compact model, which takes into account the quantum effect in planar and doublegate structures has been developed. Although our model is physically appropriate, in principle, for double-gate structure with thick silicon region and planar structure, it gives reasonable results even for DGFET with the silicon thickness of $5 \mathrm{~nm}$. The value of fitting parameter $\alpha$ of 0.5 allows to obtain good agreement with the numerical quantum device simulators in the wide range of device parameters. The quantum correction for the lateral field gives better agreement with density-gradient simulation without additional fitting parameter.

\section{Acknowledgements}

This work was supported by the NCRC program of the KOSEF through the NSI at Seoul National University, by Samsung Electronics Company and by NEDO project.

\section{References}

[1] M. J. van Dort, et.al., Sol. St. Electron., vol. 37, pp. 411-414, Mar. 1994.

[2] R. Rios, et.al., IEDM Tech. Dig., pp. 937-940, Dec. 1995.

[3] G. Baccarani and S. Reggiani, IEEE Trans. Electron Devices, vol. 46, pp. 1656-1666, 1999.

[4] G. Mugnaini, et.al. IEEE Trans. Electron Devices, vol. 52, pp. 1802-1806, 2005.

[5] C.-K. Park, et.al., IEEE Trans. Electron Devices, vol. 38, pp. 399-406, 1991.

[6] J. R. Brews, Sol. St. Electron., vol. 21, pp. 345-355, 1978.

[7] Silvaco ATLAS User's Manual

[8] L. F. Register, et.al., Applied Physics Letters, vol. 74, no. 3, pp. 457-459, 1999.

[9] S.-H. Lo,et.al., IEEE Electron Device Letters, vol. 18, no. 5, pp. 209-211, May 1997.

[10] L. Chang, et.al., IEEE Trans. Electron Devices, vol. 49, no. 12, pp. 2288-2295, 2002.

[11] S. Jin, et.al., JSTS, vol. 52, no. 11, pp.2422-2429, 2006.

[12] M. Miura-Mattausch, et.al., IEEE Trans. on CAD of IC and Systems, vol. 15, pp. 1-7, 1996. 